Author(s): Lahti, Lauri

Title:

\title{
Assistive tool for collaborative learning of conceptual structures
}

Year: $\quad 2009$

Version: Post print

\section{Please cite the original version:}

Lahti, Lauri. 2009. Assistive tool for collaborative learning of conceptual structures.

Human Computer Interaction International 2009, 19-24 July 2009, San Diego, CA, USA.

P. 53-62. 978-3-642-02713-0 (printed). DOI: 10.1007/978-3-642-02713-0_6.

Note:

The final publication is available at link.springer.com

All material supplied via Aaltodoc is protected by copyright and other intellectual property rights, and duplication or sale of all or part of any of the repository collections is not permitted, except that material may be duplicated by you for your research use or educational purposes in electronic or print form. You must obtain permission for any other use. Electronic or print copies may not be offered, whether for sale or otherwise to anyone who is not an authorised user. 
Lahti, L. (2009a). Assistive tool for collaborative learning of conceptual structures. Proc. 13th Human Computer Interaction International 2009, Part III (Universal Access in Human-Computer Interaction - Applications and Services), 19-24 July 2009, San Diego, CA, USA (ed. Stephanidis, C.). LNCS 5616, Springer, 53-62. Print ISBN 978-3-642-02712-3 and Online ISBN 978-3-642-02713-0. http://link.springer.com/chapter/10.1007/978-3-642-02713-0_6

\title{
Assistive tool for collaborative learning of conceptual structures
}

\author{
Lauri Lahti \\ Helsinki University of Technology, Department of Computer Science and Engineering \\ P.O. Box 5400, FI-02015 HUT, Finland \\ e-mail: lauri lahti at hut fi
}

\begin{abstract}
There is a demand for computational methods assisting learners to generate relevant associations for current context. Many concepts in natural language have ambiguous meanings implying alternative ways to define associations for them. It is crucial to develop collaborative methods that support free experiments with promising conceptual structures in learning. Methods for evaluating these structures in respect to the person's needs are also required. We propose a new collaborative ideation scheme and based on that we have implemented an assistive tool for learning conceptual structures in a collaborative Web environment.
\end{abstract}

Keywords: online learning, collaboration, concept map, competing values framework

\section{Introduction}

Learning new conceptual structures is a strongly personal and sensitive process. A learner needs to adopt meanings of new concepts and associate them in respect to her previous knowledge. Since establishing new associations is a highly subconscious process it is hard to explain and measure its success. The skills required for building rich mental conceptual structures are critical for learning and today's increasing information flow makes these skills even more important.

Developing support for conceptual learning is favourable in many ways since linguistic abilities are essential in all social interaction and with most software user interfaces. Thus, it seems profitable to develop new collaborative platforms that address conceptual learning. New collaborative tools are needed also among people with special needs to support for example cognitive abilities and concentration.

It has been long recognized that gaining fluent skills to read and to express oneself with language are motivated by rich communication [1]. This implies that varied collaborative environments should be introduced to everyday activities of a learner. Besides face-to-face communication also web-based collaborative platforms can provide additional support for challenged learners [2]. Since interpreting and understanding natural language still mainly remains as an unsolvable computational problem, in current research it seems feasible to focus on developing support systems that enhance learning processes on general level instead of trying to mimic the evolution of learner's knowledge with vague models. General approach avoids forced learning paths and triggers that often plague educational software and instead activates learner's own motivation and inspiration.

Our new learning tool lets people to explore word associations in collaboration and thus to get unformally practiced with vocabulary and grammer. This collaborative 
learning is based on creative problem solving model which largely relies on ideation method. A concrete goal is to build a mutually agreed concept map online.

\section{Previous research}

\subsection{Conceptual structures}

In education, conceptual relationships have been widely represented with a concept map, i.e., a graph of nodes labelled with concepts that are connected with labelled directed edges depicting their relationships. Despite the broad usage of concept mapping, all the potential of this compact notation has not yet been unleashed [3].

By building and modifying a visual concept map each learner can express and reflect her own mental conceptual structures: what are the meanings for each concepts and how they are related. In addition, the process of building a concept map allows to explore alternative conceptual structures and to compare them flexibly in a constructive manner. Especially when collaborating using a shared concept map, the learners can complement each other fruitfully by providing feedback and further ideas. Also automated evaluation of built concept maps [4] and solutions addressing special needs [5] have been proposed.

However, building conceptual structures, especially in primary and special education, is often faced with confusion or lack of motivation. To address this, creative learning strategies are needed to boost creative thinking by helping the learner to get inspiration, to achieve a new perspective and to focus her attention to things that support creation of a new idea [6].

As a computational approach for semantics Gärdenfors has suggested a model of conceptual spaces for representing the meanings of different kinds of linguistic expressions [7]. In addition, Fauconnier and Turner have argued about the human talent to create great arrays of conceptual variety that can be compressed into manageable regularities and connected to large mappings [8]. Also, Gero's FunctionBehaviour-Structure model of conceptual design has offered methodology to manage with creative process [9].

\subsection{Collaboration}

Various models have been developed to explain dynamic social processes. Competing Values Framework (CVF) [10] and its variation Innovation Genome Model (IGM) [11] are used to classify collaboration patterns both on individual and organizational level (see Fig. 1). CVF was originally developed from research on the major indicator of effective organizations by asking workers to assess the relative similarity of pairs of effectiveness measures [10]. Statistical analysis of these results provided a consistent conceptualization reducing to two major dimensions. CVF has been widely accepted and adopted as an analysis tool for enhancing organization processes [12]. IGM is a more recent variation of CVF developed for understanding specifically the 
different types of innovations that exist in organizations. In brief, the models have been orginally developed for other context that education.
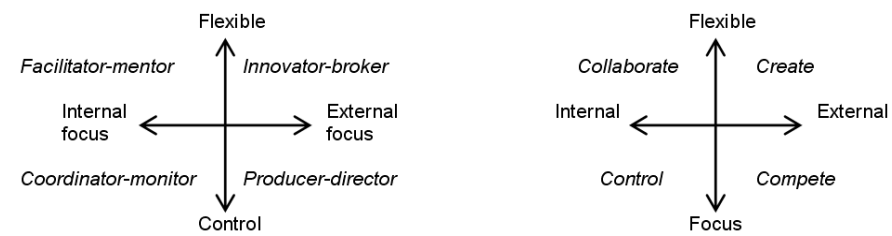

Fig. 1. (a) The Competing Values Framework and (b) the Innovation Genome Model.

Despite some differences and overlapping terminology, both CVF and IGM essentially use two dimensions that deal with internal-external orientation and flexibility-stability orientation. These two dimensions form four quadrants and both individuals and organizations can be classified to correspond one of them based on their dominant characteristics. Each quadrant represents different qualities that are present in a typical collaboration work. It has been shown that taking into account all of them enables a balanced collaboration workflow [13].

From organizational effectiveness viewpoint, each quadrant in CVF is said to represent one major model of management and to be associated with certain tasks. These models and some of their tasks include: open system model (flexibility and readiness), rational goal model (planning and goal-setting), internal process model (information management and communication) and human relations model (cohesion and morale). One variant of CVF establishes four leadership roles corresponding to previously introduced models: innovator-broker, producer-director, coordinatormonitor and facilitator-mentor. Effective managers are expected to be able to balance the competing demands belonging to all of these roles. Besides in leaders themselves, the all CVF roles can be expected to emerge in all members of a collaborating community $[14,15]$.

In IGM, each quadrant represents characteristics that produce different forms of value and innovation for specific situations. The quadrants show four organizational strengths reflecting both collective and individual practices. The quadrant names and their aims include: create (innovation and growth), compete (speed and profit), control (efficiency and quality) and collaborate (knowledge and community). It has been considered profitable to work in a group that comprises people representing all quadrants in a complementing way [11].

Both CVF and IGM have various more detailed variants and besides them there are also other more complex models concerning organizational productivity and innovation [16].

\section{Collaborative ideation scheme}

\subsection{User-initiated ideation}

We propose a new collaborative ideation scheme that is based on CVF and IGM and implemented in a platform tool. Following the models, each collaborator role is given 
to a person best matching its qualities. During ideation session the system monitors activity patterns of each role and if they differ from expected profiles more than $20 \%$ the system asks the representatives of this role to adjust that activity.

We recognize the apparent simplicity of CVF and IGM supporting our scheme and the risk of oversimplifying the complexity involved in collaboration. However, these models have established a longstanding reputation and by using them a large variety of experimental data has been produced enabling rich comparative analysis [17]. We also think that while aiming at more detailed theories about efficient collaboration it is still advisable to first start with careful low dimensional analysis. Once we have gained enough experience in our application domain with these two dimensional models we are prepared to gradually increase the amount of observable parameters.

The scheme suggests computer-assisted collaboration using following steps: formation of a group, identification of a collaborator role for each participant, sharing responsibilities according to person's collaborator role and enabling rich textual dialogue with visualizations. We see concept maps as a valuable tool to synthesize and distribute work in collaboration. The system should monitor collaboration activities and especially that personal responsibilities become fulfilled. If not, the system should provide guidance messages to restore desired activity patterns. In favour of flexible asynchronous communication, we exclude use of audio dialogue.

A key aspect of the scheme is that the participants are expected to communicate and decide themselves together about timetables, tasks to be done and division of the work. They are also responsible for themselves to really make creative efforts and to process information meaningfully since the monitoring system does not have any artificial intelligence. The system keeps track of the collaboration activities. Only if the monitored collaboration patterns greatly differ from a predefined standard flow of actions the system will intervene and give personal guidance to foster collaboration.

\subsection{Requirements for platform}

We suggest that productive computer-assisted collaboration can be based on even rather modest set of shared tools. Especially in educational domain, this ensures that complexity does not hinder intuitive usability of tools [18]. We think that computerassisted collaboration should enable participants to work at times and in locations that best suit for them, thus working asynchronously over the Web. For us it seems that a text based discussion forum supplemented with a shared concept map could serve basic communicational needs well enough for ordinary learning scenarios.

We propose that a collaborative ideation platform should provide functions to accomplish at least following tasks: to suggest new ideas accompanied with explanations, to refer to earlier suggested ideas, to comment others' ideas, to send coordination messages for selected recipients, to synthesize ideas into compact graphical notation and to distribute topics for reconsideration from graphical notation. We suggest creating a log of all actions into a database each action associated with a timestamp and contributor's name, and providing a possibility to revert back to earlier states in the ideation. It would be good to have a possibility to review filtered sets of previous actions using some criteria, like type of action or contributor. 
In our proposed scheme each collaborator gets responsibility of accomplishing tasks belonging to one quadrant of IGM closest to her. However, she may freely contribute to other tasks as well. We have tried to list some common tasks for collabaration platform that are associated with each quadrant of IGM (see Table 1). We think that tracking these tasks can enable generating automatically appropriate personal support in the proposed scheme. It needs to be emphasized that due to space constraints, our presentation here focuses only on some illustrative examples.

Table 1. Suggestion of some typical tasks for collaborator roles based on IGM.

\begin{tabular}{|l|l|l|l|}
\hline \multicolumn{1}{|c|}{ Create } & \multicolumn{1}{c|}{ Compete } & Control & Collaborate \\
\hline - submits a lot of ideas & - sets goals for ideation & - comments ideas & - aims at agreement by \\
- explores accordance of & - maintains holistic & - synthesizes ideas & personal messaging \\
ideas and concept map & efficiency & to map & - distributes topics from concept \\
- adds nodes to & - comments & - edits concept map & map for reconsideration \\
concept map & concept map & - references to ideas & - adds arcs to concept map \\
- questions constraints & - aims at logic flow & & - references to concept map \\
\hline
\end{tabular}

The collaborative ideation session is started with registration of all participants. Everyone is asked to fill in a competing values self-assessment questionnaire that is adapted from [19] and measures collaborator roles. Based on the highest ranking set of questions the most matching collaborator roles are given to participants while ensuring that each of the four roles are taken by someone. Each participant also receives a unique user account to distinguish between collaborators.

After the roles have been given, collaborators have a short warm-up. This typically includes first introducing the collaborators to each other, some social icebreaking, defining the topic of ideation and agreeing about general principles about coordination and timetable. Even if the goal may not be very clear yet, it can be practical to set some initial aims for how the ideation process can be expected to become finished.

\subsection{Activity frequency distribution}

Various working strategies have been suggested in literature for collaborative knowledge construction [20]. In the proposed scheme, a group can freely decide itself the time span for the ideation session and the timing patterns for contribution of each collaborator. A variety of time scales and activity frequency distributions can work well in collaboration [21]. We think that efficient timing practices can be adopted from real life activity patterns as people use basic functions of a collaboration platform defined above. Since personal variations can interfere measuring activity patterns experimentally we have decided to first estimate patterns analytically.

By analysing lists of typical activities identified for each collaborator role [10, 11, $22,23,24]$ we propose heuristically coarse frequency distributions for some activities performed with a collaboration platform (see Table 2). These relative frequencies try to loosely indicate how some activities are expected to be performed more by certain collaborator roles than by others. For example, persons having a Control role would refer to earlier suggested ideas on average four times more often than persons having a Compete role. Naturally, the proposed frequencies can only modestly approximate the real context-dependent activity patterns but we suggest that they can serve as a 
starting point for further elaboration. Due to space constraints, we can show here only some examples. Experimental testing is needed to acquire the real frequency values.

Table 2. Some approximated relative activity frequencies for each collaborator role.

\begin{tabular}{|l|c|c|c|c|}
\hline & Create & Compete & Control & Collaborate \\
\hline Submits ideas & 0,40 & 0,10 & 0,20 & 0,30 \\
\hline Adds nodes to concept map & 0,40 & 0,30 & 0,10 & 0,20 \\
\hline Adds arcs to concept map & 0,20 & 0,10 & 0,30 & 0,40 \\
\hline Makes references to ideas & 0,30 & 0,10 & 0,40 & 0,20 \\
\hline Makes references to concept map & 0,10 & 0,30 & 0,20 & 0,40 \\
\hline Comments ideas & 0,10 & 0,20 & 0,40 & 0,30 \\
\hline Comments concept map & 0,30 & 0,40 & 0,10 & 0,20 \\
\hline Sends coordination messages & 0,10 & 0,40 & 0,20 & 0,30 \\
\hline Synthesizes ideas to concept map & 0,20 & 0,10 & 0,40 & 0,30 \\
\hline $\begin{array}{l}\text { Distributes topics from concept map } \\
\text { for reconsideration }\end{array}$ & 0,10 & 0,20 & 0,30 & 0,40 \\
\hline $\begin{array}{l}\text { Explores accordance of ideas } \\
\text { and concept map }\end{array}$ & 0,40 & 0,30 & 0,20 & 0,10 \\
\hline Requests stimulation for creative thinking & 0,10 & 0,40 & 0,30 & 0,20 \\
\hline
\end{tabular}

\section{Prototype of platform}

\subsection{Overview}

We have implemented the proposed collaborative ideation scheme in a web-based prototype application with Java. Fig. 2 shows an overview of the user interface.

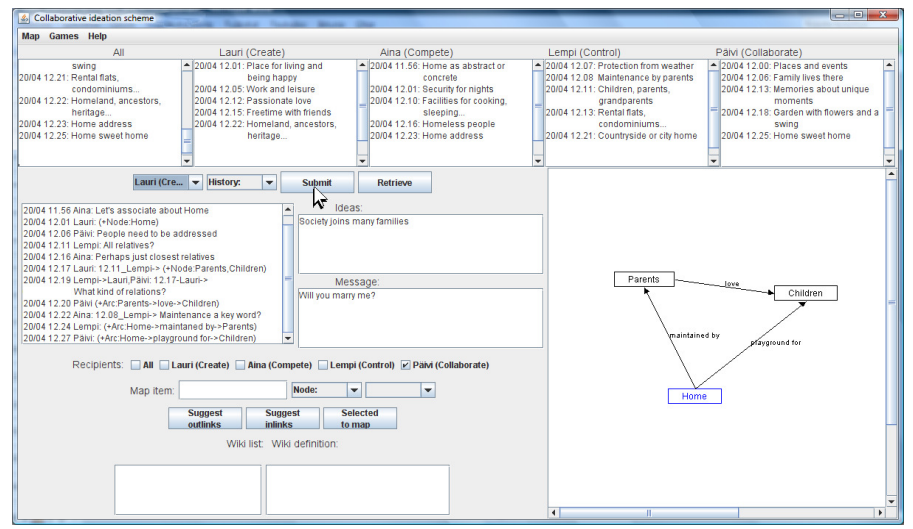

Fig. 2. User interface of the prototype.

After registration and warm-up (see chapter 3.2) the application proceeds to the actual collaborative ideation session that consists of user-initiated literal and graphical dialogue that is systematically stored into a database. Aim is to explore word associations through dialgoue and to synthesize newly learned structures to a mutually agreed concept map. The dialogue is performed in a spontaneous order but the system monitors that the activity frequencies for each collaborator role defined above are met. This should ensure most productive collaboration. If a frequency becomes 
departed over $20 \%$ from the suggested value for some collaborator role and activity, the system sends an automated message to the representatives of this role asking them to adjust that activity. If the situation does not change after three reminders the system sends a notice also to other collaborators. All collaborators are encouraged to take part in all activities but with a frequency most natural to their collaborator role.

The proposed scheme can be applied with any size of group. For easy illustration we introduce the scheme with four persons that conveniently match the roles of IGM. With more that four persons some collaborator roles may become shared. With less than four persons requires a person being responsible for several roles. The scheme can be applied even in single mode if a person takes each role one at time in a cycle.

\subsection{Operation}

In the current prototype each collaborator activates her own personalized view by selecting a correct user account from the User account pull-down menu. Then a message board on the left begins to show only messages aimed at her. These messages can be sent by other collaborators or generated automatically by the system.

On the top there is a row of scrollable text boxes. Each one is dedicated to show all ideas generated by one collaborator role of IGM in chronological order with time stamps. With over four persons, the roles become shared and individuals differentiated by font colors. In the leftmost text box all ideas together are shown in just one chronological list.

According to her intuition, each collaborator can publish ideas and messages by writing them into corresponding text boxes. The distinction here is that ideas box is reserved for actual new innovative knowledge concerning the chosen topic whereas message box is meant for all other communication. Thus messages include for example explanations accompanying the ideas, explanations accompanying edits in a shared concept map, comments about others' ideas or coordination about workflow. By using checkboxes the messages can be aimed at selected set of collaborators only, thus reducing excessive cognitive load in collaboration.

Besides writing, each collaborator can also build and edit a shared concept map on the drawing area. By using a specific text box and two pull-down menus new nodes and arcs can be added with appropriate labels and also existing nodes and arcs can be modified. Aim with the concept map is to form a mutually agreed compact visual synthesis about ideas expressed elsewhere in written form. In addition, based on this visualization coordination about remaining ideation work can be performed intuitively and distributed through messaging.

If one wants to comment or further elaborate something previously proposed item (idea, message, map edit etc.), it should be referenced by its unique time stamp and contributor's name. This enables the system to track relations between individual contributions and how synthesis is drawn or how topics are distributed for reconsideration. If a collaborator needs some stimulation for producing new ideas she can request a list of currently related concepts that are then retrieved from Wikipedia articles by pressing button Suggest inlinks or Suggest outlinks. Suitable concepts from the retrieved list can be then added directly to the shared concept map by pressing button Selected to map. 
Once the collaborator has finished adding text to text boxes and/or making changes in the concept map she is ready to submit her current contribution to the system and thus to share it with others. By pressing Submit button all additions and edits, both written and graphical, are saved to MySQL database in encoded text format accompanied with current timestamp and contributor's name. By pressing Retrieve button each collaborator can update the platform to show the most recent contributions in collaborative ideation. It is possible to revert back to earlier states in the ideation process by selecting appropriate state from History pull-down menu.

\section{Preliminary user tests}

We have carried out preliminary user tests with the prototype with five volunteers of varied background. These tests have indicated that the proposed scheme can support collaborative ideation and learning conceptual structures on pretty easy level. The scheme appears to combine completing strengths favourably also in special education. Due to constant need of new assistive tools for people with special needs, it seems well worth to make further research with the proposed scheme in this area.

Interesting finding was that the scheme can enhance initiative since the learning process can take various adaptive forms. If proceeding in the conceptual structures becomes blocked in one direction the tool allows flexibly to swift to a new perspective. This effectively prevents frustration that often arrives with other learning tools. Learners can also develop skills of comparing different approaches. Finding mutual understanding appears to be well supported despite of individual perspectives.

The individual strengths and cohesion of the group strongly affect the lifecycle of collaboration. Thus, it is difficult to reliably draw conclusions about success of a collaborative ideation scheme with user testing. Motivation can vary among collaborators and it can be hard to agree on goals. Personal interpretations of concepts can differ even unnoticed. This can lead to conflicts and prevent different collaborator roles to complement each other. The preliminary user tests expressed a need to find users with resembling learning aims to run coherent future experiments.

\section{Discussion - Concluding remarks}

The guidance automatically generated by a collaboration platform should enable enhancing each collaborator's creative output in accordance with the role they represent. In the future, the guidance could be extended to cover various aspects of ideation. The system could offer personal advice how to communicate most productively in the current context. This could deal with group cohesion, timing, goalorientation and distribution of tasks. Guidance could also help to elaborate other's ideas and to give feedback about them. The system could tell if immediate or postponed criticism would be needed to maintain fertile ideation process.

We are planning to perform extensive user tests that could evaluate our proposed scheme in various educational contexts. We are interested in extracting statistical and causal correlations in the activity patterns of persons representing different 
collaborator roles. Besides individual analysis, we are planning to examine interaction patterns between collaborators and how they accumulate their knowledge together. This could enable new ways to support characteristics of each pair-wise communication in a group. Identifying general principles of interaction patterns could also provide insight about evolution of ideas in dialogue threads.

One aspect of collaboration that requires specific emphasis in future systems is delivering a balanced ideation session that exploits available resources in a convergent fashion. Furthermore, forming synthesis and finding mutual agreement of ideas could be assisted by proposals initiated by the system when certain collaboration patterns indicate that time is right for that. Note however, that we do not expect the system to be able to evaluate ideas itself using any text analysis in the near future. The guidance generated by platform should be based solely on the activity patterns of collaboration. Thus collaborators should remain responsible about the factual content for the time being.

Many traditional collaborative ideation techniques have been based on following some strict rules. However this may not take well into account the constantly evolving dynamics of a group and how the goals change through intermediary steps. The proposed scheme tries to enable the creative resources of the group and its members to flexibly adapt and respond to the impulses gained in the flow of ideation. Therefore, the scheme does not give strict constraints for the group activities although it makes the process rather fuzzy. Anyway, in all creative work one needs to accept some uncertainty and leave room for spontaneity.

Present theories concerning the principles dictating the personality and collaboration are still ambiguous and thus it can be advisable not to get too fixated on any single theory that tries to explain these processes. For example neuroscience accompanied with computational simulations can possibly relatively soon verify some theories of human thinking and to disqualify some others. Thus, for time being it might be important to focus research efforts on general techniques that could hopefully be applicable what ever specific theories prove to be valid in the long run.

A breakthrough in collaboration theories might also come from finding new kind of transformations or mappings between individual patterns of ideation. Besides ideation, collaboration practices need to be explored on even wider scale. For example, domains of creative problem solving, problem-based learning and decision making can offer useful application areas for new innovative collaborative schemes.

\section{References}

1. MacWhinney, B. (ed.): The emergence of language. Mahwah, NJ: Lawrence Erlbaum Associates (1999).

2. Chou, S., Liu, C.: Learning effectiveness in web-based technology-mediated virtual learning environment. In: 38th Hawaii Int. Conf. on System Sciences (2005).

3. Bonastre, O., Pina, M.: Cognitive learning for distance education: a concept maps perspective. In: Fourth IEEE Int. Conf. on Cognitive Informatics, 228-231 (2005).

4. La Vecchia, L., Pedroni, M.: Concept maps as a learning assessment tool. Journal of Issues in Informing Science and Information Technology, 4 (2007). 
5. Blenkhorn, P., Evans, D.: Using speech and touch to enable blind people to access schematic diagrams. Journal of Network and Computer Applications 21, 17-29 (1998).

6. Hilliges, O., Terrenghi, L., Boring, S., Kim, D., Richter, H., Butz, A.: Designing for collaborative creative problem solving. In: 6th Int. Conf. on Creativity \& Cognition, 137 146 (2007).

7. Gärdenfors, P.: Conceptual spaces as a framework for knowledge representation. Mind and Matter, 2(2), 9-27 (2004).

8. Fauconnier, G., Turner, M.: The origin of language as a product of the evolution of modern cognition. In: Laks, B., et al. (eds.), Origin and Evolution of Languages: Approaches, Models, Paradigms. London: Equinox (2008).

9. Gero, J.: Design prototypes: a knowledge representation schema for design. AI Magazine 11(4): 26-36 (1990).

10. Quinn, R., Rohrbaugh, J.: A spatial model of effectiveness criteria: towards a competing values approach to organizational analysis. Management Science, 29, 363-377 (1983).

11. DeGraff J., Quinn, S.: Leading innovation: how to jump start your organization's growth engine. McGraw-Hill (2006).

12. Belasen, A., Frank, N.: Competing values leadership: quadrant roles and personality traits. Leadership \& Organization Development, 29(2), 127-143 (2008).

13. Buenger, V., Daft, R., Conlon, E., Austin, J.: Competing values in organizations: contextual influences and structural consequences, Organization Science, 7(5), 557-576 (1996).

14. Gregory, B., Harris, S., Armenakis, A., Shook, C.: Organizational culture and effectiveness: A study of values, attitudes, and organizational outcomes. Journal of Business Research, In press. doi:10.1016/j.jbusres.2008.05.021

15. Yang, O., Shao, Y.: Shared leadership in self-managed teams: a competing values approach. Total Quality Management, 7(5), 521-534 (1996).

16. Quinn, R., Cameron, K., Degraff, J., Thakor, A.: Competing values leadership: creating value in organizations. Edward Elgar Publishing (2006).

17. Kalliath, T., Bluedorn, A., Gillespie, D.: A confirmatory factor analysis of the competing values instrument. Educational and Psychological Measurement, 59(1), 143-58 (1999).

18. Cheon, J., Grant, M.: A cognitive load approach to metaphorical interface design: Reconsidering theoretical frameworks. In: Society for Information Technology and Teacher Education Int. Conf., 1054-1059. Chesapeake, VA: AACE (2008).

19. Quinn, R., Faerman, S., Thompson, M., McGrath, M.: Becoming a master manager: a competency framework. John Wiley \& Sons (1990).

20. Suthers, D.: Collaborative knowledge construction through shared representations. In: 38th Hawaii Int. Conf. on System Sciences (2005).

21. Stahl, G.: Supporting group cognition in an online math community: a cognitive tool for small-group referencing in text chat. Journal of Educational Computing Research, 35(2), 103-122 (2006).

22. Carte, T., Chidambaram, L., Becker, A.: Emergent leadership in self-managed virtual teams - A longitudinal study of concentrated and shared leadership behaviors. Group Decision and Negotiation 15: 323-343 (2006).

23. Pounder, J.: A behaviourally anchored rating scales approach to institutional selfassessment in higher education. Assessment \& Evaluation in Higher Education, 25(2), 171$182(2000)$.

24. Noypayak, W., Speece, M.: Tactics to influence subordinates among Thai managers. Journal of Managerial Psychology, 13(5/6), 343-358 (1998). 